\title{
Food insecurity among people who inject drugs in Athens, Greece: a study in the context of ARISTOTLE programme
}

\author{
Vana Sypsa, Eleni Flounzi, Sotirios Roussos, Angelos Hatzakis and Vassiliki Benetou* (1) \\ Department of Hygiene, Epidemiology and Medical Statistics, School of Medicine, National and Kapodistrian \\ University of Athens, 75 Mikras Asias Street, 11527 Athens, Greece
}

Submitted 21 April 2020: Final revision received 7 0ctober 2020: Accepted 16 October 2020: First published online 26 0ctober 2020

\begin{abstract}
Objective: To measure the prevalence of food insecurity and explore related characteristics and behaviours among people who inject drugs (PWID).

Design: Cross-sectional analysis of a community-based programme for HIV infection among PWID (ARISTOTLE programme). Food insecurity was measured by the Household Food Insecurity Access Scale. Computer-assisted interviews and blood samples were also collected.

Setting: A fixed location in Athens Metropolitan Area, Greece, during 2012-2013. Participants: In total, 2834 unique participants with history of injecting drug use in the past 12 months were recruited over four respondent-driven sampling rounds (approximately 1400/round).

Results: More than $50 \%$ of PWID were severely or moderately food insecure across all rounds. PWID were more likely to be severely food insecure if they were older than 40 years [adjusted OR (aOR): 1·71, 95\% CI: 1·33-2.19], were women (aOR: 1.49, $95 \%$ CI: 1.17-1.89), from Middle East countries (aOR $v$. from Greece: 1.80, $95 \%$ CI: 1·04-3.11), had a lower educational level (primary or secondary school $v$. higher education; aOR: 1.54, $95 \%$ CI: 1.29-1.84), had no current health insurance (aOR: 1.45, 95\% CI: 1.21-1.73), were homeless (aOR: 17.1, $95 \%$ CI: 12.3-23.8) or were living with another drug user (aOR: 1.55, 95\% CI: 1.26-1.91) as compared with those living alone or with family/friends. HIV-infected PWID were more likely to be severely food insecure compared with uninfected $(59.0 \% v .51 .0 \%$, respectively, $P=0.002$ ); however, this difference was attributed to the confounding effect of homelessness.

Conclusions: Moderate/severe food insecurity was a significant problem, reaching $>50 \%$ in this sample of PWID and closely related to socio-demographic characteristics and especially homelessness.
\end{abstract}

\author{
Keywords \\ Food insecurity \\ Prevalence \\ HIV infection \\ Greece \\ ARISTOTLE programme
}

Injecting drug use is a major contributor to the global burden of HIV and hepatitis $\mathrm{C}$ and $\mathrm{B}$, through the usage of contaminated injecting equipment ${ }^{(1)}$. In 2015, approximately 15.6 million people, aged 15-64 years, injected drugs globally $^{(2)}$. People who inject drugs (PWID) comprise a vulnerable population in high risk of poverty, unemployment, homelessness, incarceration and sex work, all of which are associated with increased blood-borne virus transmission $^{(2,3)}$.

Food insecurity is an important social and public health problem, especially at times of declining public health spending and economic recession. Food insecurity, defined as having uncertain or limited availability of nutritionally adequate or safe food, or the inability to acquire personally acceptable foods in socially acceptable ways, leads to diminished physical and mental health status and negatively affects social and psychological wellbeing ${ }^{(4)}$. Food insecurity is more frequent among individuals with low income, lack of a stable shelter, unemployed or in social welfare, with a history of illegal drug use and mental health problems ${ }^{(3)}$.

Prevalence of food insecurity among PWID ranges between $30 \%$ and $70 \%$, although evidence is rather limited $^{(3,5,6)}$. Food insecurity may contribute to unsafe injection practices and interferes with access to health and social support programmes for PWID, thus increasing the risk of HIV transmission ${ }^{(7)}$. Food insecurity has also been associated with reduced antiretroviral treatment effectiveness 
and suboptimal treatment adherence ${ }^{(8,9)}$. On the other hand, HIV/AIDS can contribute to food insecurity through the debilitation of the most productive household members, the decrease of individual and household economic capacity and the increase of caregiver burden ${ }^{(9)}$. Data from resource-rich settings indicate a high prevalence of food insecurity among HIV-positive individuals ${ }^{(10-12)}$, as well as among HIV-HCV co-infected populations ${ }^{(13,14)}$.

The aim of the current study was to estimate the prevalence of food insecurity among PWID living in Athens Metropolitan Area in Greece and to explore its potential association with characteristics and behaviours of this population as well as HIV infection.

\section{Materials and methods}

The ARISTOTLE programme is a 'Seek-Test-Treat' intervention programme, implemented during 2012-2013 among PWID in Athens, Greece, during an HIV outbreak in this population ${ }^{(15)}$. Details on the aim, study design, methodology and population characteristics have been described elsewhere ${ }^{(16-18)}$. In brief, ARISTOTLE was a communitybased programme aiming to decrease HIV transmission among PWID by implementing a model of care that involved reaching out to high-risk, hard to reach PWID ('seek'), engaging them in HIV testing ('test'), and initiating HIV care, opioid substitution treatment and antiretroviral therapy ('treat'). The programme has been implemented by the National and Kapodistrian University of Athens in collaboration with the Greek Organisation Against Drugs and approved by the Institutional Review Board of the Medical School of the University.

\section{Recruitment}

Recruitment was performed using respondent-driven sampling (RDS). RDS begins with a limited number of initial recruits (seeds) and uses a coupon referral scheme that requests individuals to draw from their existing social networks to identify potential recruits ${ }^{(19,20)}$. ARISTOTLE was implemented in five consecutive RDS sampling rounds with a short break in between. Recruitment target was approximately 1400 PWID per sampling round. Each round lasted 10-12 weeks with 1 week to 1 month interval between the rounds. PWID could participate in multiple rounds, but only once in each round. Eligibility criteria were (a) presentation of a valid coupon, (b) injection of drugs without a prescription in the past 12 months, (c) residing in Athens metropolitan area, (d) age $\geq 18$ years and (e) the ability to complete the interview in Greek or with the help of cultural mediators. As the questionnaire was introduced in the second sampling round, we analysed data from rounds 2 to 5 . For PWID who participated in multiple rounds, we used their first visit with available food insecurity data.

\section{Measurement of food insecurity}

The Housebold Food Insecurity Access Scale (HFIAS) a validated, widely used scale was implemented. The HFIAS was developed in the context of the US Agency for International Development's Food and Nutrition Technical Assistance project and has been applied in numerous countries around the world, particularly in resource-constrained settings $^{(21)}$. HFIAS consists of nine questions that cover three main domains of the experience of food insecurity: (a) anxiety and uncertainty about food supply, (b) insufficient quality and variety of food and (c) insufficient food intake and its consequences. Each of the nine questions ask whether a specific condition associated with the experience of food insecurity ever occurred during the previous 4 weeks and then how often this reported condition occurred during that period. The HFIAS was translated in Greek, administered and scored according to standard guidelines. The scale can take scores from 0 to 27 , with higher scores reflecting more severe food insecurity. The respondents were assigned to one of the following four categories based on a specific categorisation scheme $\mathrm{s}^{(21)}$ as follows: (1) food secure, (2) mildly food insecure, (3) moderately food insecure and (4) severely food insecure.

\section{Collection of other data}

A computer-assisted interview, administered by trained personnel, was employed using a structured questionnaire with sections on socio-demographic characteristics, injection and sexual behaviours, access to prevention and treatment ${ }^{(16)}$. Blood samples were also collected, and HIV tests were performed with a microparticle EIA anti-HIV-1/2 (AxSYM HIV-1/ $2 \mathrm{gO}, \mathrm{Abbott}$ ). HIV-1 and HIV-2 infection was confirmed by Western Blot (MP Diagnostics).

\section{Statistical analysis}

Descriptive statistics estimating median, 25th and 75th percentiles of food insecurity scores were obtained for each sampling round. Wilcoxon rank-sum test was used to assess whether there was a statistically significant change in the scores of the second and the fifth round (December 2012-March 2013 v. September-December 2013). We obtained crude (sample) as well as RDS-weighted (population) estimates for the prevalence of the various levels of food insecurity (food secure, mild, moderate, severe food insecurity) by sampling round with RDS Analysis Tool (RDSAT) $^{(22)}$.

We further explored which factors were associated with the presence of severe food insecurity among PWID. Crude and adjusted OR of severe food insecurity along with 95\% CI were obtained using logistic regression. In multivariable analysis, we accounted for correlation between recruiter and recruits due to the RDS sampling using generalised estimating equations logistic regression. We created a variable indicating who the recruiter of each subject was and used this as a cluster variable in the generalised estimating equations 
Table 1 Food insecurity score (median, 25th and 75th percentile) and distribution (numbers and percentage) of people who inject drugs (PWID) in each category of the food insecurity scale by sampling round: the ARISTOTLE programme

\begin{tabular}{|c|c|c|c|c|c|c|c|c|}
\hline \multirow[b]{2}{*}{ Food insecurity scale } & \multicolumn{2}{|c|}{ 2nd round } & \multicolumn{2}{|c|}{ 3rd round } & \multicolumn{2}{|c|}{ 4th round } & \multicolumn{2}{|c|}{5 th round } \\
\hline & $n$ & $\%$ & $n$ & $\%$ & $n$ & $\%$ & $n$ & $\%$ \\
\hline Number of participants & \multirow{2}{*}{\multicolumn{2}{|c|}{$\begin{array}{c}1429 \\
\text { December } \\
\text { 2012-March } \\
2013\end{array}$}} & \multirow{2}{*}{\multicolumn{2}{|c|}{$\begin{array}{c}1428 \\
\text { March-June } \\
2013\end{array}$}} & \multicolumn{2}{|c|}{1407} & \multirow{2}{*}{\multicolumn{2}{|c|}{$\begin{array}{c}1397 \\
\text { September- } \\
\text { December } 2013\end{array}$}} \\
\hline Time period & & & & & Sept & 2013 & & \\
\hline Median score & \multirow{2}{*}{\multicolumn{2}{|c|}{$\begin{array}{l}10 \\
0.19\end{array}$}} & \multirow{2}{*}{\multicolumn{2}{|c|}{$\begin{array}{c}9.5 \\
0,18\end{array}$}} & \multirow{2}{*}{\multicolumn{2}{|c|}{$\begin{array}{c}9 \\
0,18\end{array}$}} & \multirow{2}{*}{\multicolumn{2}{|c|}{$\begin{array}{c}7 \\
0,18\end{array}$}} \\
\hline 25th, 75th percentile & & & & & & & & \\
\hline Severely food insecure category ${ }^{*}$ & 777 & $46 \cdot 9$ & 694 & $41 \cdot 7$ & 691 & 41.4 & 627 & $43 \cdot 5$ \\
\hline Moderately food insecure category ${ }^{*}$ & 144 & $12 \cdot 0$ & 155 & 9.9 & 139 & $13 \cdot 3$ & 143 & $10 \cdot 5$ \\
\hline Mildly food insecure category & 57 & 4.5 & 109 & $7 \cdot 7$ & 97 & $4 \cdot 2$ & 109 & 4.9 \\
\hline Food secure category ${ }^{*}$ & 451 & $36 \cdot 6$ & 470 & $40 \cdot 6$ & 480 & $41 \cdot 1$ & 518 & $41 \cdot 1$ \\
\hline
\end{tabular}

*RDS-weighted proportions.

algorithm. An exchangeable correlation structure within each cluster was assumed. Multivariable analysis also accounted for differential recruitment effectiveness by HIV status and for the differing sample inclusion probabilities. We calculated inverse probability weights based on individualised recruitment weights and included them as covariate in the logistic regression models. These weights combine the individualised degree component (the inverse of the network size of the participant) with an adjustment for differential recruitment ${ }^{(23)}$, and were derived via RDSAT. We also adjusted for the different rounds of the programme by including an indicator variable in the model. Covariates included age (three categories: 18-30 years, 31-40 years and > 40 years), gender (female $v$. male), country of origin (Greek, Iran/Iraq/Afghanistan/ Pakistan and other), education (high school or higher $v$. primary school and middle/secondary school together), current living status (living without a drug user, living with a drug user and homeless), current health insurance (yes $v$. no), main substance use (Heroin/Thai, Cocaine, Speedball and Other), antiHIV status (positive $v$. negative), use of drugs divided with a syringe that someone else had already injected with in past 12 months (never, rarely/about half the time and most of the time/always) and round of ARISTOTLE programme.

\section{Results}

Data on HFIAS were available for ARISTOTLE participants from the second until the fifth sampling round, and more specifically, from 1429 PWID in the second round, 1428 in the third round, 1407 in the fourth round and 1397 in fifth round. Over the four sampling rounds, 2834 unique participants (men and women) were recruited.

Table 1 presents the median, 25th and 75 th percentile of the food insecurity score in each sampling round, as well as the distribution of participants in each category of the food insecurity scale. Across all rounds, more than $50 \%$ of PWID were severely or moderately food insecure, whereas $<1 / 3$ (37\%-41\% per round) were food secure. An improvement was observed in the median total food insecurity score in the 5 th round as compared with the second round ( $7 v .10, P<0.001)$.

Based on the data collected in all rounds of first participation, the highest proportion of severe food insecurity was identified among PWID from Iran/Iraq/Afghanistan/ Pakistan (81.3\%) and homeless (92.7\%) as presented in Table 2 .

Based on univariable analysis (Table 2), higher risk of severe food insecurity was identified among PWID aged >40 years, women, from Iran/Iraq/Afghanistan/Pakistan or other countries, with low educational level, living with drug users or homeless (as compared with PWID living alone or with family/friends), with heroin as main use of substance, with HIV infection, as well as those who used drugs divided with a used syringe. HIV-infected PWID were more likely to be severely food insecure as compared with uninfected PWID (59.0\% v. 51.0\%, respectively, $P=0.002$ ).

In the multivariable analysis, PWID had higher risk of severe food insecurity if they were $>40$ years [compared with 18-30 years; adjusted OR (aOR): 1.71, 95\% CI: 1.33-2.19], were women [aOR: 1·49, 95\% CI:1·17-1.89], from Iran/Iraq/Afghanistan/Pakistan compared with PWID from Greece [aOR: 1·80, $95 \%$ CI: 1·04-3·11], had a lower educational level (primary or secondary school $v$. higher education; aOR: 1.54, $95 \%$ CI: 1.29-1.84), were living with another drug user (aOR: $1 \cdot 55,95 \%$ CI: 1·26-1.91) or were homeless (aOR: 17.1, $95 \%$ CI: 12.3-23.8), compared with living alone or with family/friends and had no current health insurance (yes $v$. no insurance; aOR: 1.45, 95\% CI: 1.21-1.73) (Table 2). Use of drugs divided with a syringe that someone else had already injected with rarely or about half of the times ( $v$. never) in the past 12 months and use of heroin as main substance use ( $v$. cocaine) were both associated with higher risk of being severely insecure. HIV status was no longer statistically significant. The association identified in the univariable 
Table 2 Severe food insecurity according to selected characteristics for people who inject drugs (PWID) at their first participation to ARISTOTLE programme $(n=2834)$ along with crude and adjusted odds ratios $(\mathrm{OR})$ and $95 \%$ confidence intervals $(95 \% \mathrm{Cl})$ for the risk of being severely insecure

\begin{tabular}{|c|c|c|c|c|c|c|}
\hline \multirow[b]{2}{*}{ Baseline characteristics } & \multicolumn{2}{|c|}{$\begin{array}{l}\text { Severe } \\
\text { food } \\
\text { insecurity }\end{array}$} & \multirow{2}{*}{$\begin{array}{l}\text { Crude } \\
\text { OR }\end{array}$} & \multirow[b]{2}{*}{$95 \% \mathrm{Cl}$} & \multirow{2}{*}{$\begin{array}{l}\text { Adjusted } \\
\text { OR }^{*}\end{array}$} & \multirow[b]{2}{*}{$95 \% \mathrm{Cl}$} \\
\hline & $n$ & $\%$ & & & & \\
\hline \multicolumn{7}{|l|}{ Age (years) } \\
\hline $18-30$ & 338 & $51 \cdot 2$ & 1.00 & & 1.00 & \\
\hline $31-40$ & 678 & 48.2 & 0.88 & $0.74,1.07$ & 1.11 & $0.89,1.38$ \\
\hline$>40$ & 459 & 60.5 & 1.46 & $1.18,1 \cdot 80$ & 1.71 & $1.33,2 \cdot 19$ \\
\hline \multicolumn{7}{|l|}{ Gender } \\
\hline Men & 1204 & $51 \cdot 0$ & 1.00 & & 1.00 & \\
\hline Women & 271 & $58 \cdot 3$ & 1.34 & $1 \cdot 10,1 \cdot 64$ & 1.49 & $1 \cdot 17,1.89$ \\
\hline \multicolumn{7}{|l|}{ Country of origin } \\
\hline Greece & 1201 & $49 \cdot 6$ & 1.00 & & 1.00 & \\
\hline Iran/Iraq/Afghanistan/Pakistan & 100 & $81 \cdot 3$ & 4.43 & $2 \cdot 79,7 \cdot 01$ & 1.80 & $1.04,3.11$ \\
\hline Other & 174 & $62 \cdot 1$ & 1.67 & $1 \cdot 30,2 \cdot 16$ & 1.00 & $0.73,1.37$ \\
\hline \multicolumn{7}{|l|}{ Education } \\
\hline Primary school, Middle/Secondary school & 940 & $58 \cdot 4$ & 1.78 & $1.53,2.07$ & 1.54 & $1.29,1.84$ \\
\hline High School or higher & 526 & $44 \cdot 1$ & 1.00 & & 1.00 & \\
\hline \multicolumn{7}{|l|}{ Current living status } \\
\hline Living alone or with family/friends & 602 & $36 \cdot 9$ & 1.00 & & 1.00 & \\
\hline Living with a drug user & 287 & $51 \cdot 2$ & 1.79 & $1 \cdot 48,2 \cdot 18$ & 1.55 & $1.26,1.91$ \\
\hline Homeless & 581 & 92.7 & 21.6 & $15 \cdot 8,29 \cdot 7$ & $17 \cdot 1$ & $12 \cdot 3,23 \cdot 8$ \\
\hline \multicolumn{7}{|l|}{ Currently health insurance } \\
\hline Yes & 430 & $40 \cdot 4$ & 1.00 & & 1.00 & \\
\hline No & 1042 & $59 \cdot 3$ & $2 \cdot 15$ & $1.84,2.51$ & 1.45 & $1.21,1.73$ \\
\hline \multicolumn{7}{|l|}{ Main substance of use } \\
\hline Heroin/Thai† & 1220 & $54 \cdot 8$ & 1.73 & $1.41,2 \cdot 13$ & 1.44 & $1.14,1.82$ \\
\hline Cocaine & 185 & $41 \cdot 2$ & 1.00 & & 1.00 & \\
\hline Speedball & 49 & $45 \cdot 4$ & 1.19 & $0.78,1.81$ & $1 \cdot 15$ & $0.70,1.90$ \\
\hline Other & 17 & 51.5 & 1.52 & $0.75,3.08$ & 1.93 & $0.90,4 \cdot 16$ \\
\hline \multicolumn{7}{|l|}{ Anti-HIV } \\
\hline Negative & 1230 & $51 \cdot 0$ & 1.00 & & 1.00 & \\
\hline Positive & 245 & $59 \cdot 0$ & $1 \cdot 38$ & $1 \cdot 12,1.71$ & 0.98 & $0.76,1.28$ \\
\hline \multicolumn{7}{|c|}{$\begin{array}{l}\text { Use drugs divided with a syringe that someone else had already injected with } \\
\text { (past } 12 \text { months) }\end{array}$} \\
\hline Never & 905 & $49 \cdot 0$ & 1.00 & & 1.00 & \\
\hline Rarely, About half the time & 531 & $57 \cdot 9$ & 1.43 & $1 \cdot 22,1.68$ & 1.28 & $1.06,1.55$ \\
\hline Most of the time, Always & 36 & $65 \cdot 5$ & 1.98 & $1.13,3.47$ & 1.64 & $0.85,3.20$ \\
\hline \multicolumn{7}{|l|}{ Round } \\
\hline round 2 (Dec2012-Mar2013) & 787 & $54 \cdot 7$ & 1.00 & & 1.00 & \\
\hline round 3 (Mar-Jun2013) & 297 & $49 \cdot 0$ & 0.80 & $0.66,0.96$ & 0.77 & $0.61,0.96$ \\
\hline round 4 (Jun-Sep2013) & 224 & 51.6 & 0.88 & $0.71,1 \cdot 10$ & 0.83 & $0.65,1.07$ \\
\hline round 5 (Sep-Dec2013) & 167 & 48.0 & 0.76 & $0.60,0.97$ & 0.67 & $0.51,0.89$ \\
\hline
\end{tabular}

${ }^{*}$ Accounting for correlation between recruiter and recruits, differential recruitment effectiveness by HIV status and differing sample inclusion probabilities. †Thai: mix of cheap heroin and sedatives.

analysis was mainly attributed to the confounding effect of homelessness; $35.9 \%$ of HIV-infected individuals were homeless as compared with $19.8 \%$ of uninfected.

The improvement in food insecurity across rounds was confirmed in the multivariable analysis; compared with the participants of the second round (December 2012-March 2013), participants of the third and fifth rounds (MarchJune 2013 and Sep-Dec 2013, respectively) had 23\% and $33 \%$ lower risk of being severely food insecure (OR [95\% CI]: 0.77 [0.61-0.96] and 0.67 [0.51-0.89], respectively).

\section{Discussion}

In this sample of PWID living in Athens, Greece, the prevalence of moderate or severe food insecurity was high and exceeded $50 \%$. Being a female, older than 40 years, homeless or living with a drug user, from Middle East countries, having a lower educational level and no health insurance coverage were all associated with higher odds of being severely food insecure. Levels of severe food insecurity were higher among HIV-positive PWID compared with HIV-negative ones, but after adjusting for homelessness, HIV status was not associated with severe food insecurity.

Our findings are in accordance with previous studies reporting high levels of food insecurity among PWID in economically developed countries ${ }^{(5,6)}$. In a cross-sectional study conducted among 777 PWID from Los Angeles and San Francisco, USA, over half of the participants (58\%) reported food insecurity (measured by the US Adult Food Security Survey ten-item Module), while $41 \%$ met USDA criteria for very low food security status ${ }^{(6)}$. Food 
insecurity was associated with being homeless, engaging in distributive syringe sharing and feeling at risk for arrest for possession of drug paraphernalia but not with age, gender, ethnicity or race. In the context of the Vancouver Injection Drug Users Study, conducted among HIV-negative PWID, $64.7 \%$ of the participants experienced hunger and were unable to afford enough food ${ }^{(5)}$.

Women were more likely to be food insecure compared with men. Gender inequalities have been reported by other studies also in which women were the most affected individuals maybe because of gender discrimination, poverty and lack of support as head of a household ${ }^{(24)}$.

Lower education, lack of health insurance and homelessness, all indicators of lower socio-economic status, were associated with higher food insecurity risk. Of note is the particularly high risk of food insecurity among homeless PWID. Serious limitation of homeless individuals to store and preserve food, prepare proper meals and their associated stress have been proposed to explain this association $^{(5,10,11)}$. In other studies, severe food insecurity was positively associated with current living status and particularly with homelessness and living with a drug user, which both have been associated with increased probability of acquiring or transmitting $\mathrm{HIV}^{(3,6,7)}$ and may partly explain the close link observed between food insecurity and HIV in other populations ${ }^{(4,25,26)}$. In our population, severe food insecurity was more prevalent among HIV-infected individuals, mainly due to the fact that they were more likely to be homeless. This finding has serious implications among people living with HIV since food insecurity has been associated with non-adherence to antiretroviral treatment as well as reduced antiretroviral treatment effectiveness ${ }^{(8,9,27)}$. PWID reporting sharing a syringe rarely to about half of the times had $28 \%$ higher odds of being severe food insecure as compared with those who never practiced this behaviour (OR: 1.28 ; $95 \% \mathrm{CI}$ : 1.06-1.55). This difference was not significant for PWID that always shared the syringe compared with those who never, in the multivariable analysis, most probably due to the small number of people reporting always dividing drugs with a used syringe. This association has also been reported before ${ }^{(3,10)}$.

The proposal of a conceptual framework that could elucidate the links between food insecurity and injecting drug use would be useful. The framework proposed by Weiser and colleagues for food insecurity and HIV/AIDS ${ }^{(28)}$ provides some further insight, since similarities with food insecurity among PWID are evident. Thus, socio-economic factors at the community and household level, such as poverty, lack of education or access to information, as well as social factors, such as gender inequalities, drug-user related stigma and lack of social support, may influence food insecurity among PWID.

Among the limitations of our study is the cross-sectional design, which does not allow drawing conclusions upon causality of the identified associations. The questionnaire used for recording food insecurity measures access to food which is only one of the dimensions of food insecurity, thus not fully capturing the extent and magnitude of food security $^{(29)}$. Also, it has not been formally validated in the Greek population, although it is widely used in international environments. Among the strengths of the current study is that, to our knowledge, it is the first one that investigates food insecurity and its risk factors among PWID in Greece, recruited through a community-based program during an ongoing HIV outbreak. Furthermore, although access to PWID is very difficult and almost precludes a random sampling, the population coverage of the ARISTOTLE programme is estimated to be high, reaching $72 \%$ (based on the number of participants in rounds $2-5$ and the official capture-recapture estimate for the size of the PWID population in Athens), allowing us to claim that our findings represent a large portion of the specific population ${ }^{(17)}$. More affluent PWID are probably less likely to participate to a similar programme; however, RDS makes it possible to identify a hard to reach population in the absence of a sampling frame and in particular the population most in need (active PWID with no access to services).

In conclusion, severe and moderate food insecurity was recorded in more than $50 \%$ of the population of PWID participating in the current study, while several sociodemographic and other characteristics were associated with its existence and severity. Although there are no available data on food insecurity in more recent years in this population, homelessness in a sample of PWID recruited using the same methodology in 2018-2019 remains as high as in $2012-2013(27 \cdot 2 \% v \cdot 23 \cdot 1 \% \text {, respectively })^{(16,30)}$. In addition, services providing food to homeless people (including PWID) were affected during the COVID-19 pandemic when stringent social distancing measures were implemented. As food insecurity seems to consist a substantial problem among PWID, implementation of focused strategies in order to improve food access and housing of PWID, especially among women, should be among the priorities of an effective health policy planning.

\section{Acknowledgements}

Acknowledgements: N/A. Financial support: ARISTOTLE programme was supported by the National Strategic Reference Framework 2007-2013 and co-funded by the European Social Fund and national resources. Additional financial support was provided by the Hellenic Scientific Society for the study of AIDS and STD and the project 'Preventing HIV Transmission by Recently-Infected Drug Users' (NIH National Institute of Drug Abuse, National Institutes of Health; grant DP1 DA034989). Conflict of interest: The paper is submitted by Vassiliki Benetou, Associate Editor at Public Health Nutrition. Authorship: A.H. and V.S. were principal investigators of ARISTOTLE programme. V.B. conceived the idea of assessing food insecurity among PWID, translated the questionnaire in Greek and trained 
the interviewers for data collection. V.B. and V.S. drafted the manuscript. E.F. and S.R. performed statistical analysis. All authors interpreted the data and provided critical revision of the manuscript. Ethics of human subject participation: "This study was conducted according to the guidelines laid down in the Declaration of Helsinki, and all procedures involving research study participants were approved by the "The Institutional Review Board of Medical School of the National and Kapodistrian University of Athens". Written informed consent was obtained from all subjects/patients.'

\section{References}

1. Degenhardt L, Charlson F, Stanaway J et al. (2016) Estimating the burden of disease attributable to injecting drug use as a risk factor for HIV, hepatitis C, and hepatitis B: findings from the Global Burden of Disease Study. Lancet Infect Dis 16, 1385-1398.

2. Degenhardt L, Peacock A, Colledge S et al. (2017) Global prevalence of injecting drug use and sociodemographic characteristics and prevalence of HIV, HBV, and HCV in people who inject drugs: a multistage systematic review. Lancet Glob Health 5, e1192-e1120

3. Strike C, Rudzinski K, Patterson J et al. (2012) Frequent food insecurity among injection drug users: correlates and concerns. BMC Public Health 8, 1058.

4. ACC/SCN (2001) Nutrition and HIV/AIDS. Nutrition Policy Paper No. 20. Geneva: ACC/SCN.

5. Anema A, Wood E, Weiser SD et al. (2010) Hunger and associated harms among injection drug users in an urban Canadian setting. Subst Abuse Treat Prev Policy 26, 20.

6. Schmitz J, Kral AH, Chu D et al. (2016) Food insecurity among people who inject drugs in Los Angeles and San Francisco. Public Health Nutr 19, 2204-2212.

7. Weiser SD, Leiter K, Bangsberg DR et al. (2007) Food insufficiency is associated with high-risk sexual behavior among women in Botswana and Swaziland. PLoS Med 4, 1589-97.

8. Weiser SD, Frongillo EA, Ragland K et al. (2008) Food insecurity is associated with incomplete HIV RNA suppression among homeless and marginally housed HIV-infected individuals in San Francisco. J Gen Intern Med 24, 14-20.

9. Gillespie S \& Kadiyala S (2005) HIV/AIDS and Food and Nutrition Security: from Evidence to Action. Washington, DC: International Food Policy Research Institute.

10. Weiser SD, Bangsberg DR, Kegeles S et al. (2009) Food insecurity among homeless and marginally housed individuals living with HIV/AIDS in San Francisco. AIDS Behav 13, 841-848.

11. Normen L, Chan K, Braitstein P et al. (2005) Food insecurity and hunger are prevalent among HIV-positive individuals in British Columbia, Canada. J Nutr 135, 820-825.

12. Logie $\mathrm{CH}$, Wang $\mathrm{Y}$, Marcus $\mathrm{N}$ et al. (2018) Factors associated with the separate and concurrent experiences of food and housing insecurity among women living with HIV in Canada. AIDS Behav 22, 3100-3110.

13. McLinden T, Moodie EEM, Harper S et al. (2018) Injection drug use, food insecurity, and HIV-HCV co-infection: a longitudinal cohort analysis. AIDS Care 10, 1322-1328.
14. Cox J, Hamelin A-M, McLinden T et al. (2017) Food insecurity in HIV-hepatitis C virus co-infected individuals in Canada: the importance of co-morbidities. AIDS Behav 21, 792-802.

15. Paraskevis D, Nikolopoulos G, Tsiara C et al. (2011) HIV-1 outbreak among injecting drug users in Greece, 2011: a preliminary report. Euro Surveill 16, 19962.

16. Hatzakis A, Sypsa V, Paraskevis D et al. (2015) Design and baseline findings of a large-scale rapid response to an HIV outbreak in people who inject drugs in Athens, Greece: the ARISTOTLE programme. Addiction 110, 1453-67.

17. Sypsa V, Psichogiou M, Paraskevis D et al. (2017) Rapid decline in HIV incidence among persons who inject drugs during a fast-track combination prevention program after an HIV outbreak in Athens. J Infect Dis 215, 1496-1505.

18. Sypsa V, Paraskevis D, Malliori M et al. (2015) Homelessness and other risk factors for HIV infection in the current outbreak among injection drug users in Athens, Greece. $A m J$ Public Health 105, 196-204.

19. Heckathorn D (1997) Respondent-Driven sampling: a new approach to the Study of Hidden Populations. Soc Prob 44, 1741-1799.

20. Salganik MJ \& Heckathorn D (2004) Sampling and estimation in hidden populations using respondent-driven sampling. Socio Meth 34, 193-239.

21. Coates J, Swindale A \& Bilinsky P (2007) Housebold Food Insecurity Access Scale (HFIAS) for Measurement of Household Food Access: Indicator Guide. Vol. 3. Washington DC: Food and Nutrition Technical Assistance project, Academy for Educational Development.

22. Volz E, Wejnert C, Cameron C et al. (2012) RespondentDriven Sampling Analysis Tool (RDSAT), Version 7.1. Ithaca, NY: Cornell University.

23. Heckathorn DD (2007) Extensions of respondent-driven sampling: analyzing continuous variables and controlling for differential recruitment. Soc Methodol 37, 151-207.

24. Anema A, Vogenthaler N, Frongillo EA et al. (2009) Food insecurity and HIV/AIDS: current knowledge, gaps, and research priorities. Curr HIV/AIDS Rep 6, 224-231.

25. The World Bank (2007) HIV/AIDS, Nutrition, and Food Security: What We Can Do. A Synthesis of International Guidance. Washington, DC: The World Bank.

26. Pellowski JA, Kalichman SC, Cherry S et al. (2016) The daily relationship between aspects of food insecurity and medication adherence among people living with HIV with recent experiences of hunger. Ann Behav Med 50, 844-853.

27. United Nations World Food Programme (2008) HIV, food security, and nutrition. Policy Brief 2008. http://one.wfp.org/ food_aid/doc/JC1515-Policy_Brief_Expanded.pdf (accessed October 2011).

28. Weiser SD, Young SL, Cohen CR et al. (2011) Conceptual framework for understanding the bidirectional links between food insecurity and HIV/AIDS. Am J Clin Nutr 94, 1729S1739 S.

29. Ashby S, Kleve S, McKechnie R et al. (2016) Measurement of the dimensions of food insecurity in developed countries: a systematic literature review. Public Health Nutr 16, $2887-2896$

30. Sypsa V, Kalamitsis G, Paraskevis D et al. (2019) THU-427-A community-based fast-track seek-test-treat program to enhance diagnosis and linkage to care for hepatitis $\mathrm{C}$ infection among people who inject drugs in Athens, Greece (ARISTOTLE HCV-HIV program). J Hepatol 70, E344-E345. 